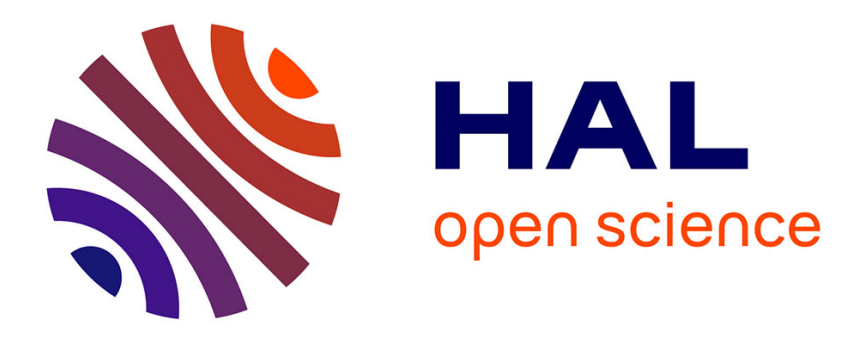

\title{
A constrained discrete layer model for heat conduction in laminated composites
}

Monique Blanc, Maurice Touratier

\section{To cite this version:}

Monique Blanc, Maurice Touratier. A constrained discrete layer model for heat conduction in laminated composites. Computers \& Structures, 2005, 83, pp.1705-1718. 10.1016/j.compstruc.2005.02.007 . hal-00019247

\section{HAL Id: hal-00019247 https://hal.science/hal-00019247}

Submitted on 18 Mar 2018

HAL is a multi-disciplinary open access archive for the deposit and dissemination of scientific research documents, whether they are published or not. The documents may come from teaching and research institutions in France or abroad, or from public or private research centers.
L'archive ouverte pluridisciplinaire HAL, est destinée au dépôt et à la diffusion de documents scientifiques de niveau recherche, publiés ou non, émanant des établissements d'enseignement et de recherche français ou étrangers, des laboratoires publics ou privés. 


\title{
A constrained discrete layer model for heat conduction in laminated composites
}

\author{
M. Blanc, M. Touratier * \\ LMSP, UMR 8106, CNRS-ENSAM-ESEM, ENSAM, 151 bd de l'Hôpital, 75013 Paris, France
}

This paper presents a new approximated computational model for heat conduction problems in laminates and is called "constrained discrete layer model". The approach is based on the well known layer-wise theory in which con-straints from continuity conditions on normal heat flux at interfaces of a multilayered structure are incorporated into the formulation in order to reduce the number of unknowns. The discrete layer method starts by discretizing the temperature field from Lagrange interpolations, in a manner of the finite element method along the thickness of the layer. The novelty in respect to discrete layer methods first introduced in solid mechanics, then comes from the exact satisfaction of all boundary and interface conditions for temperature and heat flux, using a standard variational method to express the corresponding boundary value method. The advantage is in reducing the number of unknown functions. In this paper, all the computations from both the discrete layer and constrained discrete layer models are achieved by using quadratic Lagrange interpolations in the thickness direction of the layers. This constitutes the only approximation made. Exact solutions known in the literature have been calculated here for reference solutions. Numerical results to several problems show the efficacy of the approach on one-dimensional and two-dimensional heat conduction problems in comparison with exact calculations.

Finally, the accuracy of the proposed approach can be improved by using either interpolation of a high order, or discretizing each layer in more than one subdivision, as in a finite element method. Of course, if in-layer functions are interpolated as well, then, two and three-dimensional new solid finite elements can be constructed.

Keywords: Composite laminates; Heat conduction; Discrete layer; Constrained discrete layer

\section{Introduction}

Layered structures are increasingly used in aerospace, automotive vehicles and ships. The so-called advanced composite materials were developed as part of aerospace

\footnotetext{
* Corresponding author.

E-mail address: touratier@paris.ensam.fr (M. Touratier).
}

vehicles during the second half of last century. Nowadays, there are examples of fighter and commercial aircraft, helicopters and gliders whose structures are entirely made of composite materials. Composite constructions are multilayer produced structures, mostly made of flat and curved panels, constituted of several layers or laminae, perfectly bonded together. Other examples of layered structures of interest to the present paper are especially: thermally protected structures in 
which layers with high thermal properties are used as thermal skins; thermoplastic forming; and, of course, all laminated structures submitted to heat. Thus, temperature changes often represent a significant factor, and sometimes the predominant cause of failure of composite structures subject to severe environmental loads, see for instance [1].

It has been demonstrated by Padovan $[2,3]$ that the heat conduction problem can be exactly solved for rectangular laminated plates made of orthotropic layers, for any given temperature distribution or thermal conduction conditions prescribed at the top and bottom of the plate. Such a solution has been used for thermoelastic response in laminates $[3,1]$. The analytical treatment of transient heat conduction for one-dimensional multilayered composites with an automatic solution of the eigenvalue problem is shown in Mikhailov et al. [4]. An exact methodology for analyzing linear, one-dimensional, periodic heating-cooling problems of composites with any number of laminates is given in Han [5]. For composite panels comprising a large number of laminates, the classic method of solution of problems in unsteady conduction through layered media which are traditionally based on Green's functions, a transformation technique, or the eigenvalue approach, can be quite forbidding, and even numerical algorithms may be burdensome. Alternatively, continuum models are being developed with the aim of mimicking the thermal responses of composites by using single effective thermal properties (homogenization approach) reflective of the physical makeups of the layered media, see for example the work of Maewal et al. [6].

In laminated composite shells the assumption of linear temperature distribution through the entire shell thickness (in a manner of an equivalent single layer) is hardly adequate, see [7,8]. In Carrera [9] and Zhou et al. [10], temperature distribution through the entire plate thickness is quadratic and cubic, respectively, allowing satisfying thermal boundary conditions at the top and bottom. More recently, Carrera [11], published complete heat conduction analysis for laminates from exact solutions due to Tungikar and Rao [12] for heat conduction, and computed thermoelastic responses by using different layer-wise models. The approach where each lamina of the laminate is modelled using solid finite elements may be highly impractical and may even lead to numerical difficulties as a composite laminate may contain many individual laminas each with a thickness of under $1 \mathrm{~mm}$. These modelling practices and analysis methods either lead to inefficiency or begin to break down in extreme cases where the laminate is either very thin or very thick and containing many laminas. Accurate and efficient heat conduction studies in such applications require simpler geometric models and higher order temperature approximations in all three directions of the element. Finite element approximations for lami- nated composite applications where the temperature distribution in the shell thickness direction could be of arbitrary polynomial order $p$ have been published by Surana and Orth [13], Surana and Abusaleh [14]. Hierarchical finite element approximations have been proposed for heat conduction analyses by Surana and Orth [15] and Bose and Surana [16].

In this paper we present a new approach called "a constrained discrete layer model" in which governing equations are written for each constitutive layer while interface conditions are introduced as additional constraints. Temperature field expansion is independent in each layer, and the novelty of the approach is in prescribing exactly all the interface conditions between two adjacent layers, for both temperature and heat flux, to eliminate several unknowns. Thus, for a quadratic expansion of the temperature field throughout the thickness of each layer, this leads to only one temperature unknown per layer. Consequently, in comparison to the classical discrete layer approach which we also explain in this paper, our modelling allows reducing the number of unknowns (example: for a quadratic expansion of the temperature field in the thickness of each layer, a discrete layer modelling implies $2 N+1$ unknowns to satisfy interface continuity for the temperatures in laminates having $N$ layers, while our proposed constrained discrete layer model needs only $N$ unknowns, satisfying both interface temperature and normal heat flux continuity). The discrete layer or layer-wise approach considered in the present paper (the transverse variation of the displacement field is defined in terms of a 1-D, Lagrangian, finite element representation that automatically enforces $C^{0}$ continuity of the temperature through the thickness, resulting in transverse flux component that is piecewise continuous through the thickness) was published first in elasticity by Reddy [17], and then extended to piezoelectricity, see for example Saravanos et al. [18]. In fact, several works have demonstrated the advantages of discrete layer approaches in capturing local interlaminar and intralaminar effects in mechanics, without the difficulties met in purely three-dimensional analysis. According to the authors knowledge, the constrained discrete layer approach has not been published previously, neither in mechanics nor in heat transfer. Numerical examples given in the present paper show the efficiency of our constrained discrete layer approach on one- and twodimensional heat conduction problems in laminates, by comparison both to the exact solution and to a classic discrete layer method. In fact, the present constrained discrete layer model is developed to provide better modelling capability, because the discrete layer method is equivalent to a 3-D solid finite element approximation by taking the same interpolation functions along the three coordinates, see for example [19], while the constrained discrete model allows assuring, in addition, exactly all the interface conditions (temperature, and 
normal heat flux) which permits reducing the number of unknowns (degrees of freedom in the finite element case), without reduction of accuracy. The proposed tests will show such an advantage.

\section{Formulation of the heat conduction problem in laminates}

A laminated plate occupying the parallelopipedic domain $V$ of lateral edge $\Gamma_{\mathrm{e}}$, of constant thickness $h$ and constituted by $N$ homogeneous anisotropic layers $V^{(i)}$ is considered, see Fig. 1a. The plate is referred to an orthogonal coordinate system $R=\left\{x_{\alpha}, x_{3}\right\}$, where the $x_{\alpha}(\alpha=1,2)$ axes lie in the reference plane $A$ of the plate and $x_{3}$ is in the transverse direction. The top and bottom faces of the plate and the layer interfaces, located at $x_{3}=x_{3 \mathrm{t}}, x_{3 \mathrm{~b}}$ and $x_{3 i}(i=1, N-1)$, are denoted by $\Gamma_{\mathrm{t}}$, $\Gamma_{\mathrm{b}}$ and $A_{i}$, respectively. The temperature field in the laminate $V$ is denoted by $T\left(x_{1}, x_{2}, x_{3}, t\right)$, and the heat flux components by $q_{\mathrm{m}}\left(x_{1}, x_{2}, x_{3}, t\right)$, in respect to the above coordinate system $R$, where $t$ is the time. It is assumed that the layers have a orthotropic symmetry. Consequently, thermal conductivities $k_{\alpha \beta}(\alpha \neq \beta)$ are zero all along in this paper. Therefore, the heat conduction is

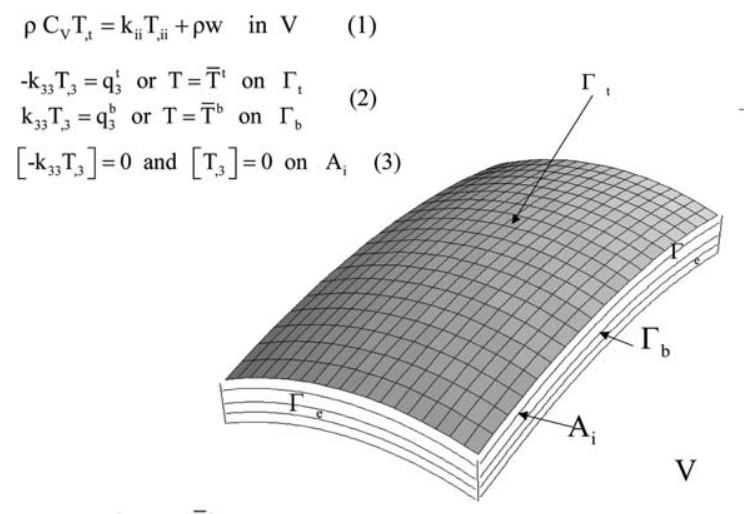

$-\mathrm{q}_{\alpha} \mathrm{n}_{\alpha}=\overline{\mathrm{q}}^{\mathrm{e}}$ or $\mathrm{T}=\overline{\mathrm{T}}^{\mathrm{e}}$ on $\Gamma_{\mathrm{e}} \quad$ (4)

(a)
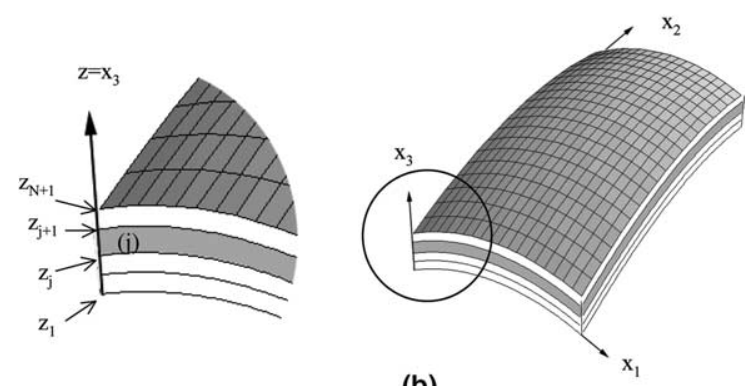

(b)

Fig. 1. (a) The composite laminate: general boundary conditions. (b) The composite laminate showing layers. The index in parentheses indicates the layer $V^{(j)}$. governed in the laminate $V$ by the following boundary-value problem for any time $t>0\left(T_{, \alpha \beta}\right.$ stands with the derivative of $T$ in respect to $x_{\alpha}$ and $x_{\beta}$, and similarly for $T_{, t}$ and $\left.T_{, 3}\right)$ :

$\rho C_{v} T_{, t}=k_{i i} T_{, i i}+\rho w$ in $V$

$\pm k_{33} T_{, 3}=\bar{q}_{3} \quad$ or $\quad T=\bar{T}^{\mathrm{b}, \mathrm{t}}$ at bottom $\Gamma_{\mathrm{b}}$, top $\Gamma_{\mathrm{t}}$

$\left[-k_{33} T_{, 3}\right]=0 ; \quad[T]=0 \quad$ at $A_{i}$

$Q=-q_{\alpha} n_{\alpha} \quad$ or $\quad T=\bar{T}^{\mathrm{e}}$ on $\Gamma_{\mathrm{e}}$

$T\left(x_{1}, x_{2}, x_{3}, 0\right)=T_{0}\left(x_{1}, x_{2}, x_{3}\right) \quad$ at time $t=0$

where $k_{i i}$ (no summation on $i$ ) are constant thermal conductivities, $\bar{T}, \bar{Q}$ and $\bar{q}_{\mathrm{m}}$ are prescribed temperatures, heat quantities and heat fluxes, respectively, $\rho$ is the mass density, $C_{v}$ is the specific heat at constant volume, and the bracket [-] denotes the jump in the enclosed argument, $n_{\alpha}$ are the in plane components of the unit normal outward to the edge $\Gamma_{\mathrm{e}}$. Note that $\rho w$ is an internal source of heat. Moreover, unless otherwise stated, throughout the paper greek and italic subscripts assume the values $(1,2)$ and $(1,2,3)$, respectively, and the summation convention over repeated indices is used. Due to the lamination of the plate, all material properties in the above equations are of course dependant on those of each layer. Solving Eqs. (1)-(5) exactly gives us the exact three-dimensional solution of the expected thermal conduction problem. Examples will be given below for several laminated plates, used to validate the new model presented in this paper.

Finally, an approximated solution of the above threedimensional problem is deduced in a classic manner either by analytical or computational approaches (finite elements for example), from a variational form of the above equations, such as:

$\forall \delta T$ such that $\delta T=0$ on $\Gamma_{T}$

$$
\begin{aligned}
\forall \tau: & \int_{0}^{\tau} \int_{V} \rho C_{V} T_{, t} \delta T \\
\mathrm{~d} V \mathrm{~d} t= & \int_{0}^{\tau} \int_{V} q_{i} \delta T_{, i} \mathrm{~d} V \mathrm{~d} t \\
& +\int_{0}^{\tau} \int_{V} \rho w \delta T \mathrm{~d} V \mathrm{~d} t \\
& +\int_{0}^{\tau} \int_{\Gamma_{Q}}\left(Q_{\Gamma}+q \cdot n\right) \delta T \mathrm{~d} \Gamma \mathrm{d} t
\end{aligned}
$$

where $Q_{\Gamma}$ is the heat quantity prescribed (natural boundary conditions) on the part $\Gamma_{Q}$ of the boundary surface $\Gamma=\Gamma_{\mathrm{t}} \cup \Gamma_{\mathrm{b}} \cup \Gamma_{\mathrm{e}}$ of the volume $V$ of the laminate, $q$ is the heat flux, $n$ is the unit normal outward from the boundary surface $\Gamma$, and $\delta T$ is the variation of the temperature $T$, at any time $t \in[0, \tau]$. Finally, $\Gamma_{T}$ is the 
complementary part of $\Gamma_{Q}$, where temperatures are prescribed (essential boundary conditions).

\section{The discrete layer model}

The laminate being subdivided into $N$ discrete layers, a continuous temperature field is assumed in each layer. The temperature field is then written in the following form:

$T\left(x_{1}, x_{2}, x_{3}, t\right)=\sum_{i=1, N} T^{(i)}\left(x_{1}, x_{2}, x_{3}=z, t\right) \chi_{z_{i}, z_{i+1}[}$

where $\chi_{z_{i}, z_{i+1}[}=1$ if $\left.z=x_{3} \in\right] z_{i}, z_{i+1}[$, and zero if not.

Introducing the nondimensionalized thickness coordinate $\xi^{i}$ for each layer $V^{(i)}$, in which $z_{i}<x_{3}=z<z_{i+1}$, see Fig. 1b,

$-1 \leqslant \xi^{i} \leqslant 1, \quad \xi^{i}=\left(z-\left(z_{i}+z_{i+1}\right) / 2\right) /\left(z_{i+1}-z_{i}\right)$

the temperature $T^{(i)}$ per layer is approximated along the thickness of the layer by quadratic Lagrange polynomial as:

$$
\begin{aligned}
& T^{(i)}\left(x_{1}, x_{2}, x_{3}=z, t\right)=\sum_{k \in\{b, m, t\}} l_{k}\left(\xi^{i}\right) T_{k}^{(i)}\left(x_{1}, x_{2}, t\right) \\
& (i=1, N)
\end{aligned}
$$

where

$$
\begin{aligned}
& l_{\mathrm{b}}(\xi)=\xi(1-\xi) / 2, \quad l_{\mathrm{m}}(\xi)=\left(1-\xi^{2}\right), \\
& l_{\mathrm{t}}(\xi)=\xi(\xi+1) / 2
\end{aligned}
$$

Thus,

$$
\begin{gathered}
T^{(i)}\left(x_{1}, x_{2}, z_{i}, t\right)=T_{\mathrm{b}}^{(i)}\left(x_{1}, x_{2}, t\right) ; \\
T^{(i)}\left(x_{1}, x_{2},\left(z_{i+1}-z_{i}\right) / 2, t\right)=T_{\mathrm{m}}^{(i)}\left(x_{1}, x_{2}, t\right) \\
T^{(i)}\left(x_{1}, x_{2}, z_{i+1}, t\right)=T_{\mathrm{t}}^{(i)}\left(x_{1}, x_{2}, t\right) \quad(i=1, \ldots, N)
\end{gathered}
$$

Prescribing

$T_{\mathrm{b}}^{(i+1)}\left(x_{1}, x_{2}, t\right)=T_{\mathrm{t}}^{(i)}\left(x_{1}, x_{2}, t\right)$

The continuity of the temperature field at the layer interfaces is then automatically satisfied. The functions $T_{j}^{(i)}\left(x_{1}, x_{2}, t\right)$ which are the unknowns of the approximated thermal conduction problem stated above, represent the temperatures of all points located on the $j$ th plane defined by $z=z_{j}$, in the laminate. They will hereafter be called "generalized temperatures". Thus, for a laminate comprising $N$ layers, such a discrete model taking a quadratic approximation through the thickness of each layer and assuring automatically the interface continuity for temperature (not for the heat flux normal to the interfaces), requires $2 N+1$ independent generalized temperatures when using only one subdivision per layer. It is of course possible to subdivide more layers to improve the accuracy of the computation. We will show this in sections devoted to numerical evaluations of the modeling. This kind of thickness discretization may be seen as a classic finite element approximation along the corresponding direction of the laminate. If we choose quadratic Lagrangian interpolation of the generalized temperatures $T_{j}^{(i)}\left(x_{1}, x_{2}, t\right)$, in each layer, then, the well known three-dimensional 27-nodes parallelopipedic finite element is recovered. In the present paper, we develop analytical solutions of composite laminated plates from the variational formulation (6), in which Eqs. (7)(12) have to be inserted.

Taking into account Eqs. (10)-(12), it is easy to show that the temperature per layer given by Eq. (9), may be written for the $i$ th layer of a laminated plate as:

$$
\begin{aligned}
T^{(i)}\left(x_{1}, x_{2}, z, t\right)= & l_{\mathrm{b}}\left(\xi^{i}\right) T_{\mathrm{b}}^{(i)}\left(x_{1}, x_{2}, t\right) \\
& +l_{\mathrm{m}}\left(\xi^{i}\right) T_{\mathrm{m}}^{(i)}\left(x_{1}, x_{2}, t\right) \\
& +l_{\mathrm{t}}\left(\xi^{i}\right) T_{\mathrm{b}}^{(i+1)}\left(x_{1}, x_{2}, t\right)
\end{aligned}
$$

Inserting this last expression in Eq. (7) of the temperature field, and substituting the result in the variational form (6) of the boundary value problem, give equations of the heat conduction problem for laminated plates as (see also Fig. 2):

$$
\begin{aligned}
\forall \delta T_{\mathrm{b}}^{(1)}\left(x_{1}, x_{2}, t\right): \quad & \int_{z_{1}}^{z_{2}}\left\{\square^{(1)}\left(T^{(1)}\left(x_{1}, x_{2}, z, t\right)\right)\right. \\
- & \left.\rho^{(1)} w^{(1)}\right\} l_{\mathrm{b}}\left(\xi^{1}\right) \mathrm{d} z=0 \\
\forall \delta T_{\mathrm{m}}^{(j)}\left(x_{1}, x_{2}, t\right): \quad & \int_{z_{j}}^{z_{j+1}}\left\{\square^{(j)}\left(T^{(j)}\left(x_{1}, x_{2}, z, t\right)\right)\right. \\
& \left.-\rho^{(j)} w^{(j)}\right\} l_{\mathrm{m}}\left(\xi^{j}\right) \mathrm{d} z=0, \quad j=1, \ldots, N
\end{aligned}
$$

$$
\begin{aligned}
& \forall \delta T_{\mathrm{b}}^{(j)}\left(x_{1}, x_{2}, t\right): \quad j=1, \ldots, N-1, \\
& \int_{z_{j}}^{z_{j+1}}\left\{\square^{(j)}\left(T^{(j)}\left(x_{1}, x_{2}, z, t\right)\right)-\rho^{(j)} w^{(j)}\right\} l_{t}\left(\xi^{(j)}\right) \mathrm{d} z \\
& \quad+\int_{z_{j+1}}^{z_{j+2}}\left\{\square^{(j+1)}\left(T^{(j+1)}\left(x_{1}, x_{2}, z, t\right)\right)\right. \\
& \left.\quad-\rho^{(j+1)} w^{(j+1)}\right\} l_{\mathrm{b}}\left(\xi^{(j+1)}\right) \mathrm{d} z=0
\end{aligned}
$$

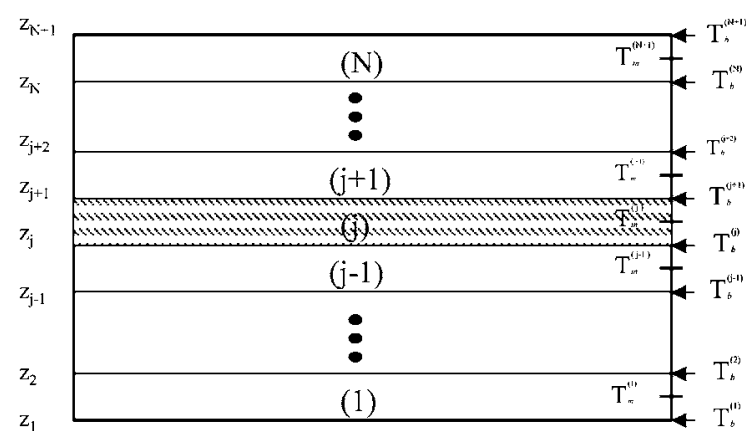

Fig. 2. Configuration of the $j$ th layer, and generalized temperatures. 


$$
\begin{aligned}
& \forall \delta T_{\mathrm{b}}^{(N+1)}\left(x_{1}, x_{2}, t\right): \quad \int_{z_{N}}^{z_{N+1}}\left\{\square^{(N)}\left(T^{(N)}\left(x_{1}, x_{2}, z, t\right)\right)\right. \\
& \left.-\rho^{(N)} w^{(N)}\right\} l_{\mathrm{t}}\left(\xi^{(N)}\right) \mathrm{d} z=0
\end{aligned}
$$

where

$$
\square^{(j)} T^{(j)}=\rho^{(j)} C_{v}^{(j)} T_{, t}^{(j)}-k_{1}^{(j)} T_{, 11}^{(j)}-k_{2}^{(j)} T_{, 22}^{(j)}-k_{3}^{(j)} T_{, z z}^{(j)}
$$

is a differential operator (this quadrilateral symbol is often used in wave propagation analysis, see for example [20]), applied here to the temperature per layer $T^{(j)}$ given by the above Eq. (13). Since all materials in each layer are assumed orthotropic for thermal conductivities, we have written $k_{i i} \equiv k_{i}$ above, and we will keep this notation hereafter throughout this paper.

From the variational form (6), natural and essential boundary conditions are written as:

$$
\int_{0}^{\tau} \int_{\Gamma_{Q}}\left(q_{i} n_{i}+Q_{\Gamma}\right) \delta T \mathrm{~d} \Gamma \mathrm{d} t=0 \forall \delta T \text { on } \Gamma_{Q}
$$

or

$$
\int_{0}^{\tau} \int_{\Gamma_{Q}}(T-\bar{T}) \delta T \mathrm{~d} \Gamma \mathrm{d} t=0 \forall \delta T \text { on } \Gamma_{T}
$$

Important remark. In the above variational formulation of the boundary value problem, and for examples shown hereafter, constant thermal conductivities have been assumed, as well as their nonzero values only for $k_{i i}=k_{i}$. This is of course not a limitation of our modelling, which has the capability to be fully general by incorporating a complete conductivity matrix, interface thermal resistance, and even including temperature dependence for thermal conductivities. In this case, the thermal boundary value problem becomes nonlinear, and has to be solved using the tangent operator technique. In addition, it is evident that this kind of modelling is fully general and has the capability to represent any type of boundary conditions concerning heat transfer.

\section{The constrained discrete layer model}

The constrained discrete layer approach starts with the above Eq. (13). The, continuity conditions at the interfaces for the heat flux are then taken into account, and corresponding equations solved in order to reduce the number of independent generalized temperatures $T_{j}^{(i)}\left(x_{1}, x_{2}, t\right)$, which occur in Eq. (13), so as to construct the temperature field as in Eq. (7). The resulting conditions at interfaces between layers $V^{(i)}$ and $V^{(i+1)}$ are therefore stated as follows:

$$
\begin{aligned}
&(-\left.k_{3}^{(i)} T^{(i)}\left(x_{1}, x_{2}, z, t\right)_{3,3}\right)_{z=z i+1} \\
& \quad=\left(-k_{3}^{(i+1)} T^{(i+1)}\left(x_{1}, x_{2}, z, t\right)_{, 3}\right)_{z=z i+1} ; \quad i=1, \ldots, N-1
\end{aligned}
$$

From Eq. (13) the above continuity conditions for the heat flux at the interfaces can be expressed as:

$$
\begin{aligned}
i=1, \ldots, N-1 & \\
4 \kappa^{(i)} T_{m}^{(i)}+4 \kappa^{(i+1)} T_{\mathrm{m}}^{(i+1)}= & \kappa^{(i+1)}\left(3 T_{\mathrm{b}}^{(i+1)}+T_{\mathrm{b}}^{(i+2)}\right) \\
& +\kappa^{(i)}\left(3 T_{\mathrm{b}}^{(i+1)}+T_{b}^{(i)}\right)
\end{aligned}
$$

with $\kappa^{(j)}=k_{3}^{(j)} /\left(z_{j+1}-z_{j}\right)$

In Eqs. (21), generalized temperatures $T_{p}^{(j)}$ depend on in plane coordinates and time, as shown in Eq. (13). Interface continuity conditions (21) then furnish a system of $N-1$ equations allowing the elimination of $T_{\mathrm{b}}^{(2)}, \ldots$, $T_{\mathrm{b}}^{(N)}$ in functions of the unknowns $T_{\mathrm{b}}^{(1)}, T_{\mathrm{m}}^{(1)}, T_{\mathrm{m}}^{(2)}$, $T_{\mathrm{m}}^{(3)}, \ldots, T_{\mathrm{m}}^{(N)}, T_{\mathrm{b}}^{(N+1)}$. Note that if boundary conditions are expressed at the top and bottom faces of the laminate, then $T_{\mathrm{b}}^{(1)}$ and $T_{\mathrm{b}}^{(N+1)}$ are prescribed as we will show below.

Finally, the temperature per layer $T^{(j)}\left(x_{1}, x_{2}, z, t\right)$, $j=1, \ldots, N$, can be written as:

$$
\begin{aligned}
T^{(j)}\left(x_{1}, x_{2}, z, t\right)= & \sum_{i=1, N}\left(L_{\mathrm{m}}^{(i)}\left(\xi^{j}, \kappa^{(1)}, \ldots, \kappa^{(N)}\right) T_{\mathrm{m}}^{(i)}\left(x_{1}, x_{2}, t\right)\right) \\
& +L_{\mathrm{b}}^{(1)}\left(\xi^{j}, \kappa^{(1)}, \ldots, \kappa^{(N)}\right) T_{\mathrm{b}}^{(1)}\left(x_{1}, x_{2}, t\right) \\
& +L_{\mathrm{b}}^{(N+1)}\left(\xi^{j}, \kappa^{(1)}, \ldots, \kappa^{(N)}\right) T_{\mathrm{b}}^{(N+1)}\left(x_{1}, x_{2}, t\right)
\end{aligned}
$$

where coefficients $L_{k}^{(n)}$ depend on Lagrange polynomial given by Eq. (10), and also, both on their first derivative and on $\kappa^{(j)}$ as defined by Eq. (22). In fact, the corresponding new shape functions $L_{k}^{(j)}\left(\xi^{j}, \kappa^{(1)}, \ldots, \kappa^{(N)}\right)$ through the thickness are quadratic $\beta$-Spline functions.

From Eqs. (6), (7) and (23), heat conduction equations for the constrained discrete layer theory are derived as

$$
\begin{aligned}
\dot{\forall} & \delta T_{\mathrm{m}}^{(r)}\left(x_{1}, x_{2}, t\right): \\
& \sum_{i=1, N} \int_{z_{i}}^{z_{i+1}}\left\{\square^{(i)} \sum_{s=1}^{N}\left(L_{\mathrm{m}}^{(s)}\left(\xi^{i}, \kappa^{(1)}, \ldots, \kappa^{(N)}\right) T_{\mathrm{m}}^{(s)}\left(x_{1}, x_{2}, t\right)\right)\right. \\
& +L_{\mathrm{b}}^{(1)}\left(\xi^{i}, \kappa^{(1)}, \ldots, \kappa^{(N)}\right) T_{\mathrm{b}}^{(1)}\left(x_{1}, x_{2}, t\right) \\
& \left.+L_{\mathrm{b}}^{(N+1)}\left(\xi^{i}, \kappa^{(1)}, \ldots, \kappa^{(N)}\right) T_{\mathrm{b}}^{(N+1)}\left(x_{1}, x_{2}, t\right)\right) \\
& \left.-\rho^{(i)} w^{(i)}\right\} L_{\mathrm{m}}^{(r)}\left(\xi^{i}\right) \mathrm{d} z=0, \quad r=1,2, \ldots, N
\end{aligned}
$$

where the differential operator $\square^{(i)}(\cdot)$ is obtained from (18).

Corresponding natural and essential boundary conditions can be expressed as follows:

$\forall \delta T\left(x_{1}, x_{2}, z, t\right)$ on $\Gamma_{\mathrm{e} Q}$ :

$$
\int_{0}^{\tau} \int_{\Gamma_{\mathrm{e} Q}}\left(q_{\alpha} n_{\alpha}+Q\right) \delta T \mathrm{~d} \Gamma \mathrm{d} t=0 \quad \text { natural }
$$


or on $\Gamma_{\mathrm{e} Q}$ :

$$
\int_{0}^{\tau} \int_{\Gamma_{\mathrm{e} T}}(T-\bar{T}) \delta T \mathrm{~d} \Gamma \mathrm{d} t=0 \quad \text { essential }
$$

because with this constrained discrete layer theory, boundary conditions on top and bottom faces of the laminate have to be prescribed in the corresponding temperature field given by substituting Eq. (23) in Eq. (7). Thus, for example, if temperatures $\bar{T}^{\text {sup }}\left(x_{1}, x_{2}, t\right)$ and $\bar{T}^{\text {inf }}\left(x_{1}, x_{2}, t\right)$ are prescribed respectively on the top and bottom faces of a laminate, then we have to write in Eq. (23):

$\bar{T}^{\text {sup }}\left(x_{1}, x_{2}, t\right)=T_{\mathrm{b}}^{(N+1)}\left(x_{1}, x_{2}, t\right)$ and
$\bar{T}^{\text {inf }}\left(x_{1}, x_{2}, t\right)=T_{\mathrm{b}}^{(1)}\left(x_{1}, x_{2}, t\right)$

Similarly, a normal heat flux on the top and bottom boundary of a laminate may be prescribed as:

$-\kappa^{(1)}\left(-3 T_{\mathrm{b}}^{(1)}+4 T_{\mathrm{m}}^{(1)}-T_{t}^{(1)}\right)=\bar{q}_{3}^{\mathrm{inf}}$

$-\kappa^{(N)}\left(T_{\mathrm{b}}^{(N)}-4 T_{\mathrm{m}}^{(N)}+3 T_{b}^{(N+1)}\right)=\bar{q}_{3}^{\text {sup }}$

which allows expressing $T_{\mathrm{b}}^{(1)}$ and $T_{\mathrm{b}}^{(N+1)}$ in functions of the prescribed fluxes $\bar{q}_{3}^{\text {inf }}$ and $\bar{q}_{3}^{\text {sup }}$. Of course, combinations of these boundary conditions are also possible.

Finally, to prescribe edge boundary conditions, the constrained discrete layer theory requires to satisfy Eqs. (25) and (26), while any top and bottom boundary conditions have to be imposed directly in Eq. (23) by using Eqs. (27)-(29). Satisfying exactly all the interface continuity conditions and top and bottom boundary conditions, the great advantage of this theory is that it implies only one independent generalized temperature per layer, if quadratic transverse interpolation of the temperature is taken in each layer.

Comments on relationship between layer-wise (discrete layer) and finite element approximations. In both discrete layer and constrained discrete layer approaches shown above, the transverse variation of the temperature field is defined in terms of a one-dimensional finite element representation. The laminate thickness dimension is subdivided into a series of one-dimensional finite elements (i.e., thickness subdivisions) whose nodes correspond to planes of constant coordinate in the undeformed laminate. The 1-D finite element mesh contains nodes distributed through the thickness, the functions $l_{k}\left(\xi^{i}\right)$ in Eq. (9) are the one-dimensional, quadratic Lagrangian interpolation functions associated with the series of nodes (or planes), distributed through the thickness of the laminate, whereas the functions $L_{k}^{(j)}$ in Eq. (23) are quadratic $\beta$-Spline functions. For example, if we choose only one subdivision in each layer, then, along the thickness of each layer we associate one one-dimensional quadratic finite element. Finally, discrete layer/constrained discrete layer approaches as described above are like one-dimensional finite element approximations along the thickness coordinate, and exact closed (analytical) form for surface coordinate temperature dependency. Thus, it is evident that with the discrete layer model, if we choose a quadratic Lagrangian approximation for the temperature field along surface coordinates, as along thickness coordinate, we recover the 3-D solid finite element discretisation based on the 27 nodes brick element, having 27 nodal degrees of freedom (nodal temperatures). Choosing the constrained discrete layer model, we can deduce a new $C^{0} 3$-D 27 nodes solid finite element (brick element) having only nine nodal degrees of freedom (nodal temperatures at mid-surface), like the nine node quadrilateral Lagrangian element.

\section{Applications and numerical evaluations}

\subsection{Stationary thermal waves in a four-layer composite panel}

Both discrete layer and constrained discrete layer theories are now evaluated from a first example provided by Han [5]. It is a composite laminated panel, layered along its transverse $z$-direction, in four layers, and indefinitely extended following the mid-plane. An exact solution has been derived from Eqs. (1)-(4). We here compare our two theories (discrete layer and constrained discrete layer) with this exact solution. All the corresponding symbolic and numerical computations for exact, discrete layer and constrained discrete layer theories were performed by using the Mathematica software, Wolfram [21].

Fig. 3 shows the composite panel with four layers of different, isotropic materials of which the $j$ th layer has constant physical properties $\rho^{(j)}, C_{v}^{(j)}$, and $k^{(j)}$, and thickness $h_{j}=z_{j+1}-z_{j}$. The boundary conditions at $z_{1}=0$ show zero heat flux $q_{3}$, and that at $z_{5}=l$ a fluctuating temperature, i.e.,

$$
\begin{aligned}
& q_{3}(z=0, t)=0 \quad \text { and } \\
& \quad T(z=l, t)=\bar{T}^{\text {sup }}(t)=T_{\mathrm{e}} \exp (\mathrm{i} \omega t)
\end{aligned}
$$

where, as in [5], we take $T_{\mathrm{e}}=1$, and $\omega$ is the circular frequency, while $\mathrm{i}\left(\mathrm{i}^{2}=-1\right)$ is the imaginary number. From Eqs. (1)-(4), the corresponding exact solution of this boundary value problem is then given by:

$$
\begin{gathered}
T^{(j)}(z, t)=\left[A_{j} \cosh \left(\beta^{(j)} z_{j}\right)+B_{j} \sinh \left(\beta^{(j)} z_{j}\right)\right] \exp (\mathrm{i} \omega t) \\
j=1, \ldots, 4
\end{gathered}
$$

in which the eigencoefficient $\beta^{(j)}$ is related to physical properties and circular frequency by:

$\beta^{(j)}=(1+i)\left(\rho^{(j)} C_{v}^{(j)} \omega /\left(2 k^{(j)}\right)\right)^{1 / 2}$

In Eq. (31), coefficients $A_{j}$ and $B_{j}$ are complex constants to be classically determined from boundary conditions and interface continuity conditions, both as regards 


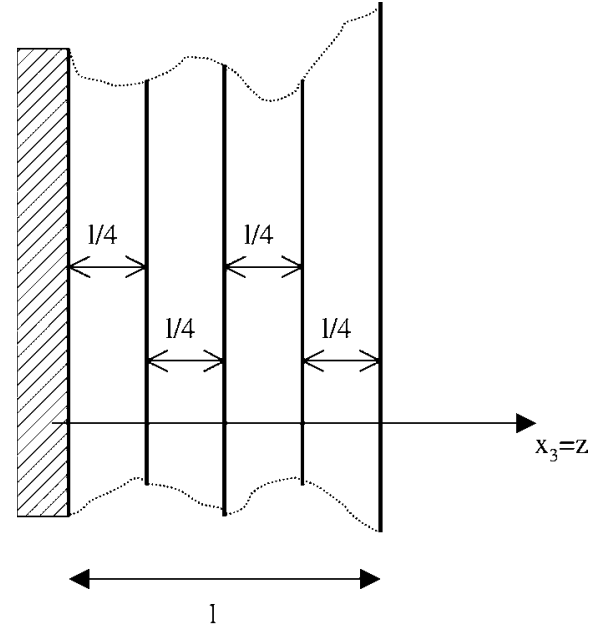

Fig. 3. Lamination scheme for the four layer panel to study stationary thermal waves.

temperature and heat flux. With the discrete layer theory, based for example on four discrete layers, equations to be solved for the present example, are Eqs. (13)-(18) plus (30), taking $j=1, \ldots, 4 ; \quad N=4 ; \quad$ and, of course, $T_{\mathrm{b} \text { or } m}^{(i \text { or }}(t)$ in Eq. (13). This give us a linear differential system of eight equations with time $t$, having to satisfy boundary conditions (30). Of course, due to the nature of this differential system, solutions as regards time have to be researched in the form of Eq. (30), i.e.: $D_{r} \exp (\mathrm{i} \omega t)$, where $D_{r}$ are complex constants, involving a linear algebraic system with complex coefficients to be solved. The discrete layer theory allows satisfying interface continuity conditions for temperature, and boundary conditions regarding temperature. In the case of the constrained discrete layer theory, corresponding equations of this simple boundary value problem have to be found from the general heat conduction variational Eq. (24) by taking $i=1, \ldots, 4 ; N=4 ; T_{\mathrm{m}}^{(s)}(t)$ in Eq. (24), and satisfying direct boundary conditions in Eqs. (27) and (28). This involves only four linear time differential equations when taking four discrete layers modelling the four-layered panel. The solution of this boundary value problem is then researched in the same form as that shown above for the discrete layer theory, the important difference being that the constrained discrete layer theory permits exactly satisfying all the interface and boundary conditions (temperature and normal flux), and needing only four degrees of freedom. Note that the discrete layer and constrained discrete layer theories are identical to their quadratic finite element approximations for this very particular case, which is reduced to a one-dimensional problem.

Computed temperature and heat flux distributions expressed in terms of real $R\left(T\right.$ or $\left.q_{3}\right)$ and imaginary $I\left(T\right.$ or $\left.q_{3}\right)$ parts are shown in Fig. 4 for $\bar{\omega}=16$, and in Fig. 5 for $\bar{\omega}=256$, where $\bar{\omega}$ is a nondimensional circular frequency expressed as in [5] by $\bar{\omega}=\omega\left(l^{2} / \alpha_{\mathrm{m}}\right)$, in which $l$ is the overall thickness of the four-layer composite, and $\alpha_{\mathrm{m}}$ the minimum diffusivity of the layers. From Ref. [5], the thermal properties of the four layers have conduction coefficient $k$ and mass heat $\rho C_{v}$ in the ratios $1: 2: 4: 8$, the thermal diffusivities being equal for all layers. It is observed that thermal waves have greater penetration depths at moderate frequencies, while they are confined near the end of the laminate at high frequencies. Both discrete layer and constrained discrete layer models are very satisfactory compared to the exact solution, using only two subdivisions in each layer. Therefore, in this last discretization, the discrete layer model implies 15 independent generalized temperatures or degrees of freedom, while the constrained discrete model needs 8 degrees of freedom to get such good results. However, using only one subdivision per layer does not produce so good results at high frequencies $(\bar{\omega}=256)$. As expected, the discontinuity of the heat flux is observed for the discrete layer model.

The next example is devoted to studying convergence velocity of our two approximate models by comparison with the exact solution, extracted from Eqs. (1)-(4).

\subsection{Convergence study of the discrete layer and constrained discrete layer models for a two-layer laminate}

In this study we consider a two layer perfectly bonded composite laminate derived from the above example, see Fig. 3. Physical properties have been changed, now to be in the ratio $\rho, C_{v}, k=1$ for the first layer (which is localized between $z=0$ and $z=1 / 2$ ), and 10 for the second one. Boundary conditions are kept as for the above problem, see Eq. (30), but layer thicknesses are $1 / 2$. Such thermal problems appear severe, as can be verified in Table 1 for temperature at the abcissa $z=0$, and in Table 2 for the heat flux at $z=l$. We analyse the convergence of the discrete layer and constrained discrete layer models in respect to the number of equal subdivisions in each layer. Real and imaginary parts of temperature calculated at the origin of the laminate, see Table 1, and those of the heat flux at the end of the laminate, see Table 2 , are compared with the corresponding values obtained from the exact analytical solution (Eqs. (1)-(4)). Results show good convergence properties towards the exact values for both the discrete layer and constrained discrete layer models. However, if we refer to the number nu of independent generalized temperatures (degrees of freedom), the constrained discrete layer model allows reaching exact values for both real and imaginary parts of temperatures and heat flux more quickly.

\subsection{Computations of heat conduction in a sandwich media}

By using both exact solutions provided by Eqs. (1)-(4), discrete layer model (Eqs. (7) and (13)-(17)), 
1 subdivision per layer

$R(\mathrm{~T})$

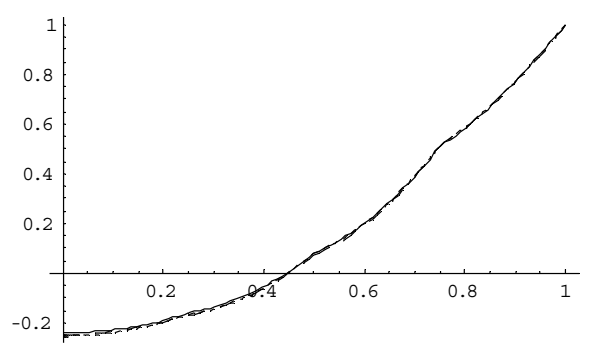

$I(\mathrm{~T})$
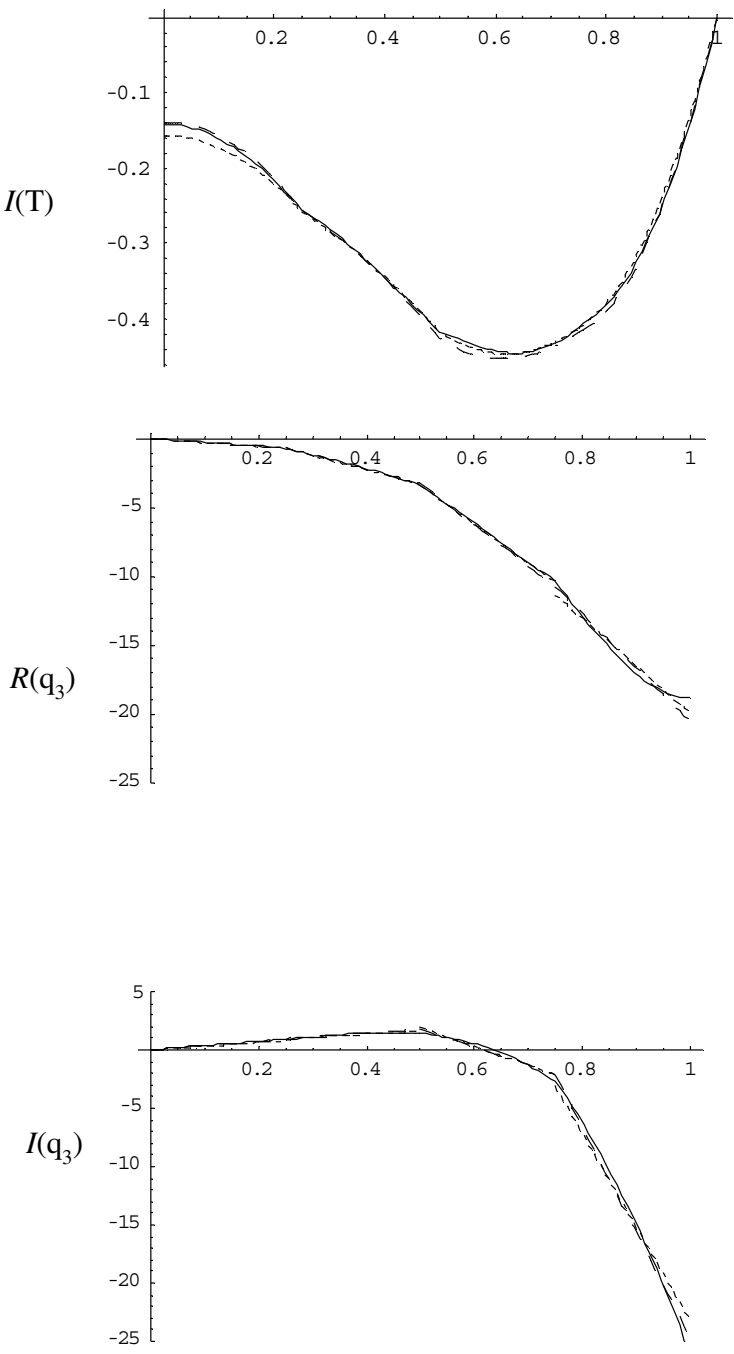

2 subdivisions per layer
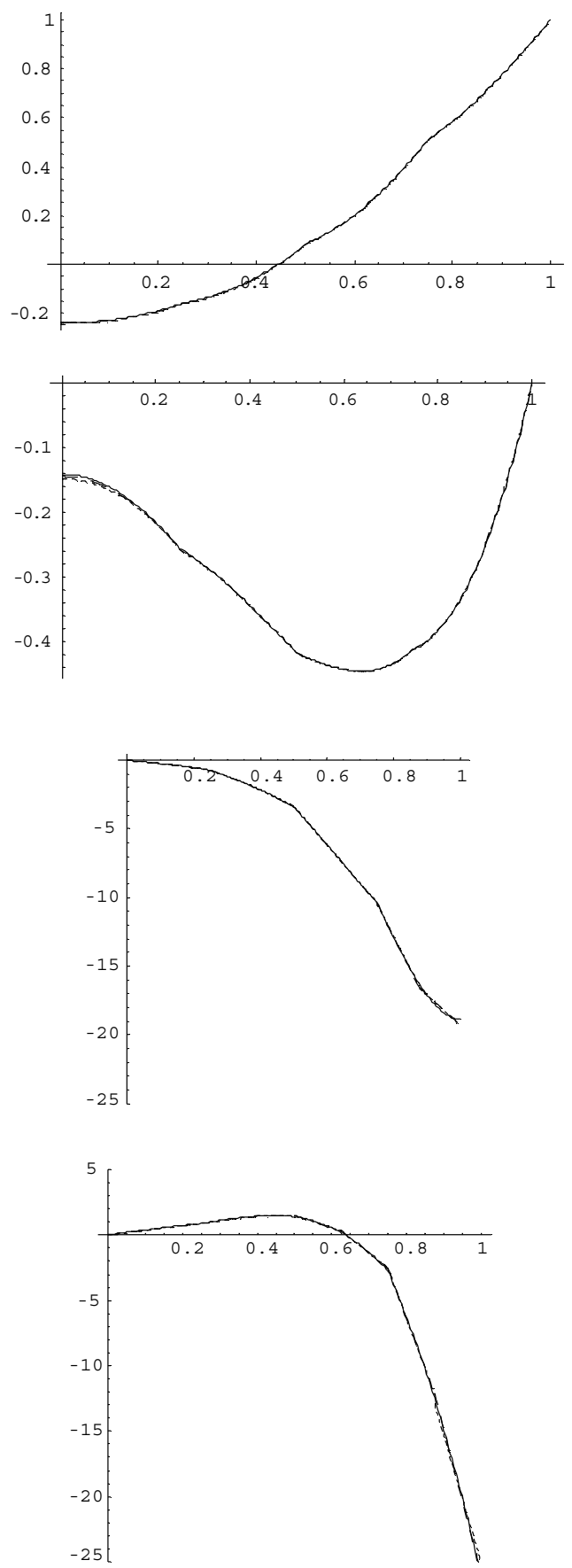

Fig. 4. Distributions of real $R(\cdot)$ and imaginary $I(\cdot)$ parts of the temperature $T$ and heat flux $q_{3}$ along the $z$-axis of the four layer panel shown in Fig. 3. Exact solution (-), discrete layer solution (..), and constrained discrete layer solution (---) are compared. On the left is shown a calculation using one subdivision per layer for both discrete layer and constrained discrete layer, and two subdivisions in each layer on the right. Reduced circular frequency is $\bar{\omega}=16$, and abscissa is $z / l$.

and constrained discrete layer model (Eqs. (7), (23), (24)), numerical calculations of temperature and heat flux are obtained for a rectangular $(l / b=2)$ symmetric sandwich media for which geometry and thermal 
1 subdivision per layer

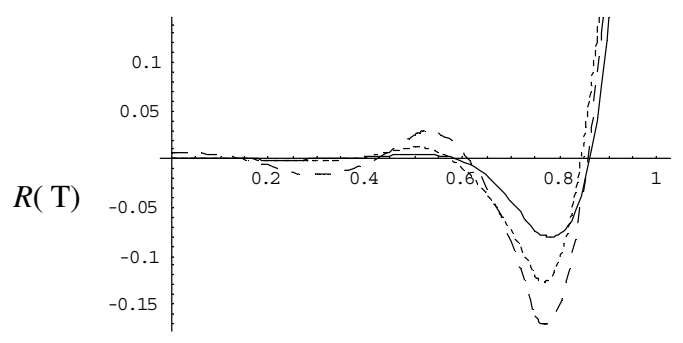

$I(\mathrm{~T})$
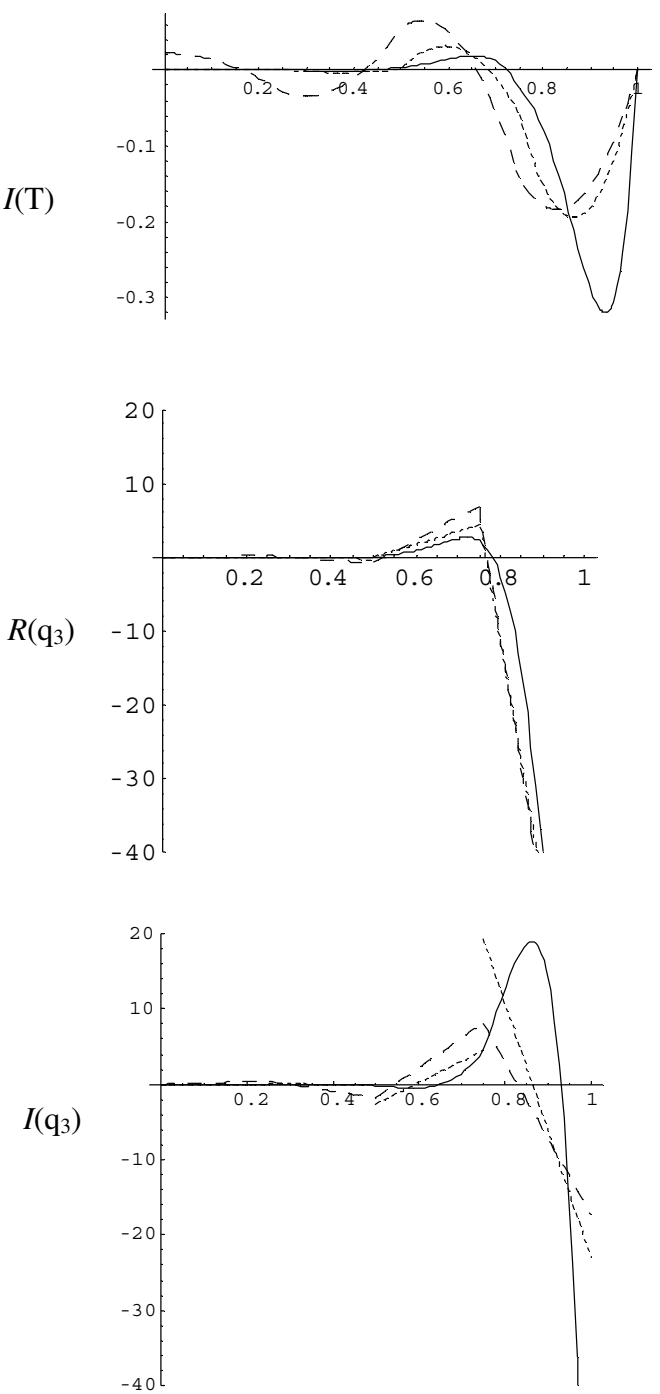

2 subdivisions per layer
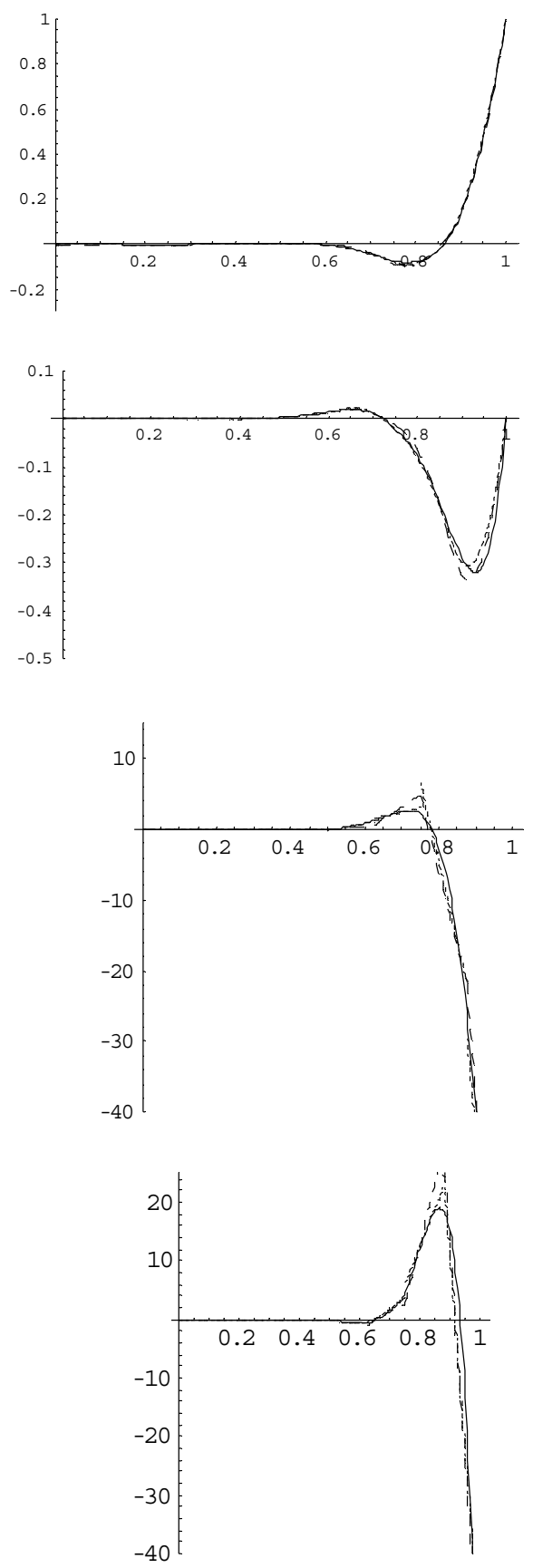

Fig. 5. Distributions of real $R(\cdot)$ and imaginary $I(\cdot)$ parts of the temperature $T$ and heat flux $q_{3}$ along the $z$-axis of the four layer panel shown in Fig. 3. Exact solution (-), discrete layer solution ( $\cdots)$, and constrained discrete layer solution (---) are compared. On the left is shown a calculation using one subdivision per layer for both discrete layer and constrained discrete layer, and two subdivisions in each layer on the right. Reduced circular frequency is $\bar{\omega}=256$, and abscissa is $z / l$.

properties, as well as boundary conditions are specified in Fig. 6. The sandwich is composed of an isotropic core of thickness $b-2 h$, and isotropic skins of equal thickness $h=0.2 b$, and is submitted to a simply sinusoïdal 
Table 1

Convergence properties in respect to the number ns of subdivisions in each layer for the discrete layer and constrained discrete layer models

\begin{tabular}{llclclcrc}
\hline $\mathrm{ns}$ & $R\left(T_{\mathrm{DL}}(0)\right)$ & $\mathrm{nu}$ & $R\left(T_{\mathrm{CDL}}(0)\right)$ & $\mathrm{nu}$ & $I\left(T_{\mathrm{DL}}(0)\right)$ & $\mathrm{nu}$ & $I\left(T_{\mathrm{CDL}}(0)\right)$ & $\mathrm{nu}$ \\
\hline 1 & -0.337 & $(3)$ & -0.265 & $(2)$ & -0.106 & $(3)$ & -0.048 & $(2)$ \\
2 & -0.237 & $(7)$ & -0.225 & $(4)$ & -0.089 & $(7)$ & -0.065 & $(4)$ \\
3 & -0.228 & $(11)$ & -0.224 & $(6)$ & -0.087 & $(11)$ & -0.073 & $(6)$ \\
4 & -0.226 & $(15)$ & -0.223 & $(8)$ & -0.085 & $(15)$ & -0.076 & $(8)$ \\
5 & -0.225 & $(19)$ & -0.223 & $(10)$ & -0.084 & $(19)$ & -0.077 & $(10)$ \\
6 & -0.224 & $(23)$ & -0.223 & $(12)$ & -0.083 & $(23)$ & -0.078 & $(12)$ \\
7 & -0.224 & $(27)$ & -0.223 & $(14)$ & -0.083 & $(27)$ & -0.078 & $(14)$ \\
8 & -0.224 & $(31)$ & -0.222 & $(16)$ & -0.082 & $(31)$ & -0.078 & $(16)$ \\
9 & -0.223 & $(35)$ & -0.222 & $(18)$ & -0.082 & $(35)$ & -0.079 & $(18)$ \\
10 & -0.223 & $(39)$ & -0.222 & $(20)$ & -0.082 & $(39)$ & -0.079 & $(20)$ \\
12 & -0.223 & $(47)$ & -0.222 & $(24)$ & -0.081 & $(47)$ & -0.079 & $(24)$ \\
15 & -0.223 & $(59)$ & -0.222 & $(30)$ & -0.081 & $(59)$ & -0.079 & $(30)$ \\
20 & -0.223 & $(79)$ & -0.222 & $(40)$ & -0.081 & $(79)$ & -0.079 & $(40)$ \\
25 & -0.222 & $(99)$ & -0.222 & $(50)$ & -0.080 & $(99)$ & -0.079 & $(50)$ \\
30 & -0.222 & $(119)$ & -0.222 & $(60)$ & -0.080 & $(119)$ & -0.079 & $(60)$ \\
& & $R\left(T_{\text {exact }}(0)\right)=-0.222$ & & & $I\left(T_{\text {exact }}(0)\right)=-0.079$ & \\
\hline
\end{tabular}

Convergence is observed on real $R(\cdot)$ and imaginary $I(\cdot)$ parts of the temperature $T(0)$ computed at the abscissa $z=0$, in the bi-layer laminate. Reference solutions are the exact values $R\left(T_{\text {exact }}(0)\right)$ and $I\left(T_{\text {exact }}(0)\right)$. Temperature computed from the discrete layer model is labelled $T_{\mathrm{DL}} ; T_{\mathrm{CDL}}$ from the constrained discrete model. In parentheses is the number nu of independent generalized temperatures (degrees of freedom) corresponding to ns subdivisions (discretization) per layer.

Table 2

Convergence properties in respect to the number ns of subdivisions in each layer for the discrete layer and constrained discrete layer models

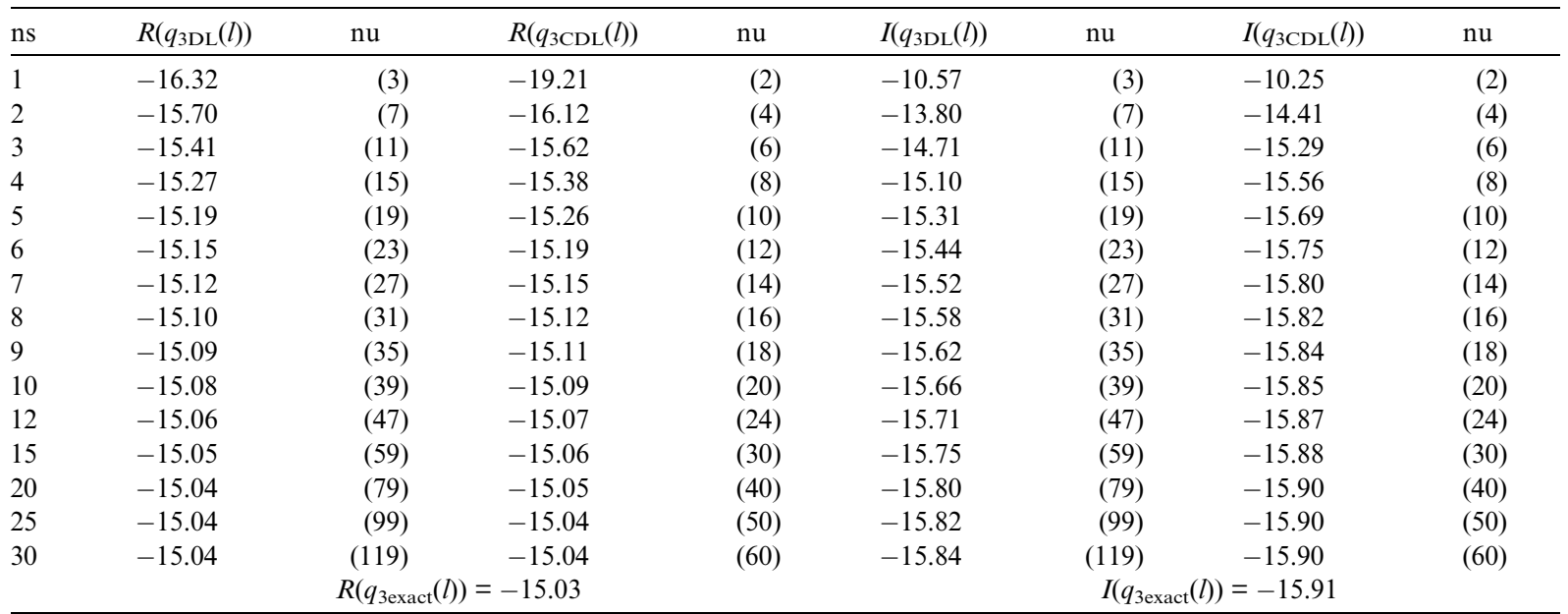

Convergence is observed on real $R(\cdot)$ and imaginary $I(\cdot)$ parts of the heat flux $q_{3}(l)$ computed at the abscissa $z=l$, in the bi-layer laminate. Reference solutions are the exact values $R\left(q_{3 \text { exact }}(l)\right)$ and $I\left(q_{3 \text { exact }}(l)\right)$. Heat flux computed from the discrete layer model is labelled $q_{3 \mathrm{DL}} ; q_{3 \mathrm{CDL}}$ from the constrained discrete model. In parentheses is the number nu of independent generalized temperatures (degrees of freedom) corresponding to ns subdivisions (discretization) per layer.

temperature loading given by $\bar{T}^{t}=T_{0} \sin \left(\pi x_{2} / l\right)$ with $T_{0}=1$ at its top edge, while zero temperature is prescribed on the other three edges. Temperature distributions along the thickness of the sandwich are given in Table 3 at station $x_{2}=l / 2$ of the cross-section of the sandwich for the exact solution $T_{\text {(exact) }}$, discrete layer calculations using one and two subdivisions per layer depict $T_{(\mathrm{DL} 1)}, T_{(\mathrm{DL} 2)}$ respectively, and constrained discrete layer computations based on one and two subdivisions per layer, as defined by $T_{(\mathrm{CDL} 1)}, T_{(\mathrm{CDL} 2)}$ respectively. From the discrete layer and constrained discrete layer models, the distribution of the components $q_{\alpha}, \alpha=2$ or 
Geometric and thermal properties of the sandwich

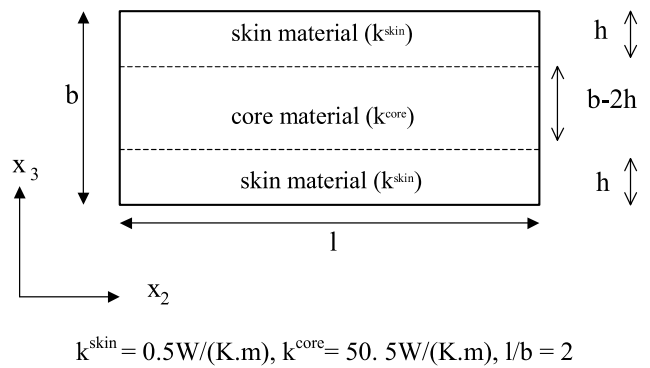

Boundary conditions prescribed at top, bottom and lateral edges of the sandwich

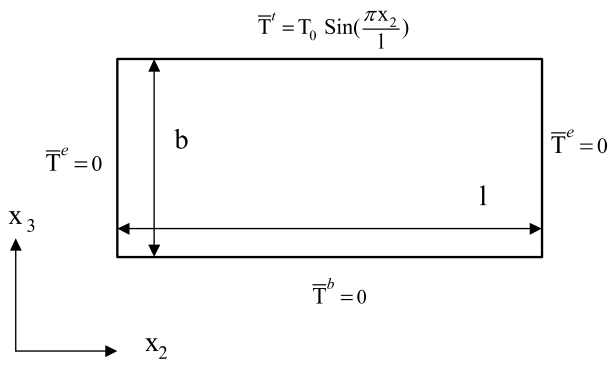

Fig. 6. Geometric and thermal properties, and thermal boundary conditions for the two-dimensional sandwich problem.

3, of the heat flux along the thickness of the sandwich media are compared with the exact calculations $q_{\alpha \text { (exact) }}$ in Table $4\left(q_{2}\right.$ component along the edge $\left.x_{2}=l\right)$ and in Table $5\left(q_{3}\right.$ component at the station $\left.x_{2}=/ / 2\right)$.

From Tables $3-5$, it is observed that both discrete layer and constrained discrete layer calculations are in good agreement with the exact solution, even when using only one subdivision in each layer. It is recalled that the discrete layer model needs six independent generalized temperatures such as $T_{\mathrm{bor} m}^{(i \text { or } i+1)}\left(x_{1}, x_{2}, t\right)$ in Eq.
(13) for one subdivision per layer, and 11 for two subdivisions per layer. The constrained discrete layer model requires only three independent generalized temperatures such as $T_{\mathrm{m}}^{(i)}\left(x_{1}, x_{2}, t\right)$ in Eq. (23) for a discretization incorporating one subdivision per layer, and only six when discretizing each layer with two subdivisions. Of course, due to both in plane and the no time dependence character of this thermal problem, $x_{1}$ and $t$ have been disregarded. Finally, the discontinuity of the component $q_{3}$ of the heat flux is seen Table 5, as expected for the discrete layer model, but not, of course, for the constrained discrete layer theory. Fig. 7 shows that those distributions are globally in very good agreement with exact calculations. However, constrained discrete layer computations need only six independent generalized temperatures, while discrete layer calculations require 11 independent generalized temperatures for two subdivisions per layer of which results are shown in Fig. 7 and Tables 3-5. These tables confirm the efficiency of the constrained discrete layer model, by comparison with the discrete layer one, in terms, both of size reduction and convergence/accuracy properties.

Remark. Note that for a medium equivalent to this shown in Fig. 6, but having isotropic material properties, it is immediate to verify that DL2 and CDL2 modelling (i.e., based on only two subdivisions through the entire thickness of the isotropic medium) suffice to get very accurate results in comparison with exact calculations. Such result would be expected regarding those obtained in every layer for a sandwich medium, using two subdivisions per layer, see for example Fig. 7.

\section{Summary and conclusions}

In this paper, a new computational model is proposed to analyze heat conduction in composite

Table 3

Distribution of the temperature $T\left(x_{2}=/ / 2, x_{3}\right) / T_{0}$ along the thickness at the station $x_{2}=l / 2$, for the composite sandwich shown in Fig. 6

\begin{tabular}{llllll}
\hline$x_{3}$ & $T_{\text {(DL1) }}$ & $T_{\text {(DL2) }}$ & $T_{\text {(exact) }}$ & $T_{\text {(CDL2) }}$ & $T_{\text {(CDL1) }}$ \\
\hline$x_{2}=1 / 2$ & & & & & 0.000 \\
0 & 0.000 & 0.000 & 0.000 & 0.013 & 0.000 \\
0.1 & 0.013 & 0.013 & 0.013 & 0.026 & 0.013 \\
0.2 & 0.026 & 0.026 & 0.026 & 0.027 & 0.026 \\
0.3 & 0.027 & 0.027 & 0.027 & 0.028 & 0.028 \\
0.4 & 0.028 & 0.028 & 0.028 & 0.030 & 0.030 \\
0.5 & 0.030 & 0.030 & 0.030 & 0.032 & 0.033 \\
0.6 & 0.032 & 0.032 & 0.032 & 0.036 & 0.036 \\
0.7 & 0.036 & 0.036 & 0.036 & 0.040 & 0.040 \\
0.8 & 0.040 & 0.040 & 0.040 & 0.514 & 1.000 \\
0.9 & 0.515 & 0.514 & 0.514 & 1.000 & 000 \\
1 & 1.000 & 1.000 & &
\end{tabular}

Notations $T_{(\mathrm{DL} j)}$ and $T_{(\mathrm{CDL} j)}$ refer to the temperatures computed from the discrete layer and constrained discrete layer models, respectively. The index $j=1,2$ is the number of subdivisions per layer; $T_{\text {(exact) }}$ is the exact value. 
Table 4

Distribution of the component $q_{2}\left(x_{2}=l, x_{3}\right)$ of the heat flux along the thickness at the station $x_{2}=l$ for the sandwich considered in Fig. 6

\begin{tabular}{llllll}
\hline$x_{3}$ & $q_{3(\mathrm{DL} 1)}$ & $q_{3(\mathrm{DL} 2)}$ & $q_{3(\mathrm{exact})}$ & $q_{3(\mathrm{CDL} 2)}$ & $q_{3(\mathrm{CDL} 1)}$ \\
\hline$x_{2}=l / 2$ & & & & & -0.065 \\
0 & -0.064 & -0.065 & -0.065 & -0.066 & -0.067 \\
0.1 & -0.066 & -0.066 & -0.066 & -0.068 & -0.065 \\
$0.2-$ & -0.068 & -0.068 & -0.068 & -0.068 & -0.063 \\
$0.2+$ & -0.001 & -0.059 & -0.068 & -0.398 & -0.063 \\
0.3 & -0.377 & -0.399 & -0.396 & -0.729 & -0.820 \\
0.4 & -0.753 & -0.738 & -0.733 & -1.059 & -1.198 \\
0.5 & -1.129 & -1.060 & -1.089 & -1.490 & -1.576 \\
0.6 & -1.504 & -1.481 & -1.472 & -1.920 & -1.954 \\
0.7 & -1.880 & -1.902 & -2.891 & -2.350 & -2.332 \\
$0.8-$ & -2.256 & -2.323 & -2.356 & -2.350 & -2.399 \\
$0.8+$ & -2.349 & -2.351 & -2.390 & -2.386 & -2.466 \\
0.9 & -2.400 & -2.391 & -2.483 & -2.478 & \\
1 & -2.451 & -2.472 & & \\
\hline
\end{tabular}

Notations $q_{2(\mathrm{DL} j)}$ and $q_{2(\mathrm{CDL} j)}$ refer to the component $q_{2}$ of the heat flux computed from discrete layer and constrained discrete layer models, respectively. The index $j=1,2$ indicates the number of subdivisions per layer, $q_{2 \text { (exact) }}$ is the exact value of the temperature.

Table 5

Distribution of the component $q_{3}\left(x_{2}=l / 2, x_{3}\right)$ of the heat flux along the thickness at the station $x_{2}=l / 2$ for the sandwich considered in Fig. 6

\begin{tabular}{|c|c|c|c|c|c|}
\hline$x_{3}$ & $q_{2(\mathrm{DL} 1)}$ & $q_{2(\mathrm{DL} 2)}$ & $q_{2 \text { (exact) }}$ & $q_{2(\mathrm{CDL} 2)}$ & $q_{2(\mathrm{CDL} 1)}$ \\
\hline \multicolumn{6}{|c|}{$x_{2}=l$} \\
\hline 0 & 0.000 & 0.000 & 0.000 & 0.000 & 0.000 \\
\hline 0.1 & 0.010 & 0.010 & 0.010 & 0.010 & 0.010 \\
\hline $0.2-$ & 0.021 & 0.021 & 0.021 & 0.021 & 0.020 \\
\hline $0.2+$ & 2.073 & 2.072 & 2.072 & 2.073 & 2.042 \\
\hline 0.3 & 2.103 & 2.108 & 2.109 & 2.109 & 2.081 \\
\hline 0.4 & 2.191 & 2.198 & 2.197 & 2.198 & 2.180 \\
\hline 0.5 & 2.339 & 2.340 & 2.340 & 2.338 & 2.339 \\
\hline 0.6 & 2.546 & 2.540 & 2.541 & 2.538 & 2.557 \\
\hline 0.7 & 2.812 & 2.805 & 2.804 & 2.806 & 2.834 \\
\hline $0.8-$ & 3.137 & 3.137 & 3.137 & 3.141 & 3.170 \\
\hline $0.8+$ & 0.031 & 0.031 & 0.031 & 0.031 & 0.032 \\
\hline 0.9 & 0.404 & 0.403 & 0.403 & 0.403 & 0.403 \\
\hline 1 & 0.785 & 0.785 & 0.785 & 0.785 & 0.785 \\
\hline
\end{tabular}

$q_{3(\mathrm{DL} j)}$ and $q_{3(\mathrm{CDL} j)}$ refer to the component $q_{3}$ of the heat flux computed from discrete layer and constrained discrete layer models, respectively. The index $j=1,2$ indicates the number of subdivisions per layer, $q_{3 \text { (exact) }}$ is the exact value of the temperature.

laminates. It is derived from the well known layer-wise/ discrete layer method applied in solids mechanics. The novelty in the present approach is in reducing the size of the model, by "a priori" enforcing interface and boundary conditions on the heat flux, in addition to those in temperatures. This allows us to derive the formulation of the thermal boundary value problem from a standard variational principle in temperature. It has been demonstrated that a quadratic expansion of the temperature field throughout the thickness of each layer of composite laminates, leads to only one independent generalized temperature per layer. Examples have been presented for one- and two-dimensional problems for composite laminates. Numerical comparisons made between exact solutions, discrete layer and constrained discrete layer models, shown the efficiency of the approach, which could be extended to anisotropic laminated threedimensional problems, interface discontinuity by incorporating thermal resistances, as well as to any kind of boundary conditions, or temperature dependency for conduction coefficients, in this last case solving a nonlinear problem. It will be easy to derive new finite elements from this formulation, having just to interpolate the independent generalized temperatures. Note that for 


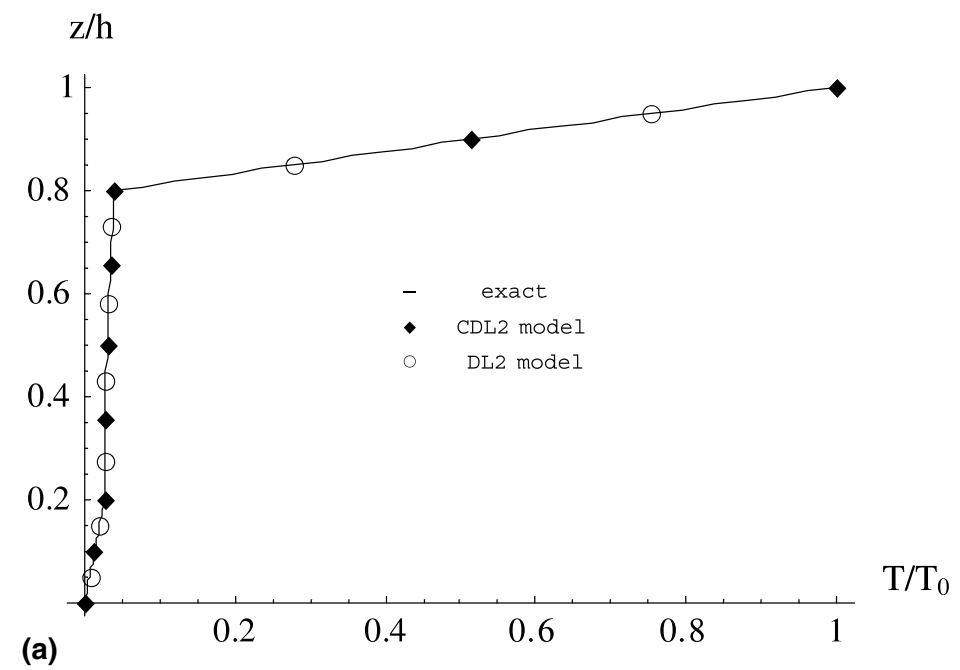

(a)

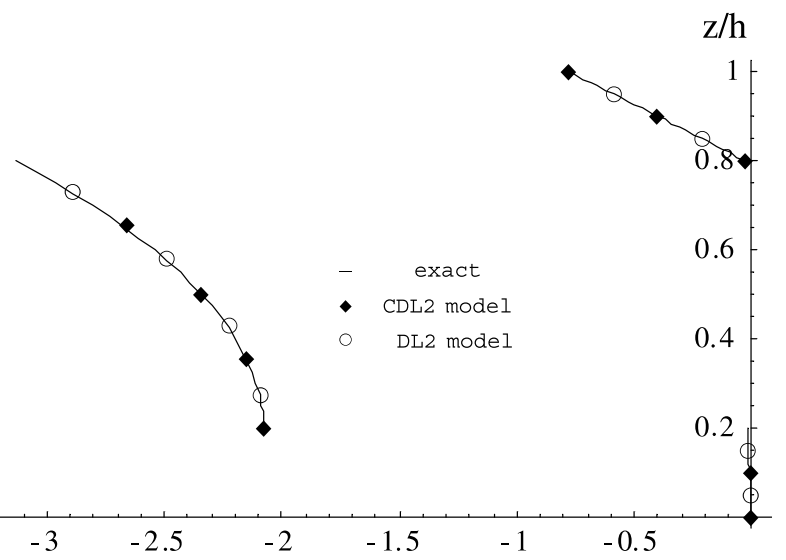

(b)

(c)

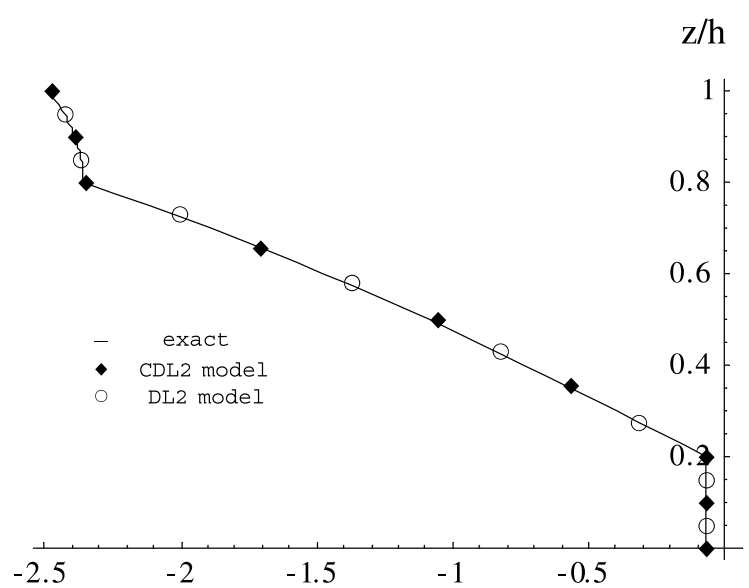

Fig. 7. Temperature profile $T\left(l / 2, x_{3}\right) / T_{0}$, (a) normal $q_{3}\left(l / 2, x_{3}\right)$, (b) and in-plane $q_{2}\left(l, x_{3}\right)$, (c) heat flux component distributions, for the sandwich shown in Fig. 6, when using two subdivisions per layer.

one-dimensional problems, our approach is actually a finite element approximation. Future papers will be de- voted to the suggested extension capabilities of the proposed modeling. 


\section{References}

[1] Savoia M, Reddy JN. Three-dimensional thermal analysis of laminated composite plates. Int $\mathbf{J}$ Solids Struct 1995;32(5):593-608.

[2] Padovan J. Solution of transient temperature fields in laminated anisotropic slabs and cylinders. Int $\mathbf{J}$ Eng Sci 1975;13:247-60.

[3] Padovan J. Thermoelasticity of anisotropic generally laminated slabs subject to spacially periodic thermal loads. ASME J Appl Mech 1975;June:341-6.

[4] Mikhailov MD, Özisik NL, Vulchanov NL. Diffusion in composite layers with automatic solution of the eigenvalue problem. Int J Heat Mass Transfer 1983;26(8):113141.

[5] Han Lit S. Periodic heat conduction through composite panels. AIAA—J Thermophys 1986;1(2):184-6.

[6] Maewal A, Bache TC, Hegemier GA. A continuum model for diffusion in laminated composite media. J Heat Transfer 1976; February:133-8.

[7] Bose A, Surana KS. Axisymmetric shell element for heat conduction in laminated composites. Comput Struct 1993;48(1):33-49.

[8] Chattopadhyay A, Li J, Gu H. Coupled thermo-piezoelectric-mechanical model for smart composite laminates. AIAA J 1999;37(12):1633-8.

[9] Carrera E. Theories for thermal stress analysis of orthotropic multilayered plates. J Thermal Stresses 2000;23: 797-831.

[10] Zhou X, Chattopadhyay A, Gu H. Dynamic responses of smart composites using a coupled thermo-piezoelectricmechanical model. AIAA J 2000;38(10):1939-48.
[11] Carrera E. Temperature profile influence on layered plates response considering classical and advanced theories. Amer Inst Aeronaut Astronaut J 2002;40(9):1885-96.

[12] Tungikar VB, Rao KM. Three dimensional exact solution of thermal stresses in rectangular composite laminate. Compos Struct 1994;27:419-30.

[13] Surana KS, Orth NJ. Elements for heat conduction in laminated composites. Comput Struct 1989;33(5):1251-65.

[14] Surana KS, Abulsaleh G. Curved shell elements for heat conduction with $p$-approximation in the shell thickness direction. Comput Struct 1990;34(6):861-80.

[15] Surana KS, Orth NJ. p-Version hierarchical three-dimensional curved shell element for heat conduction. Comput Mech 1991;7:341-53.

[16] Bose A, Surana KS. Piecewise hierarchical $p$-Version curved shell finite element for heat conduction in laminated composites. Comput Struct 1993;49(2):283-300.

[17] Reddy JN. A generalisation of two-dimensional theories of laminated composite plates. Commun Appl Numer Meth 1987;3:173-80.

[18] Saravanos DA, Heyliger PR, Hopkins DA. Layerwise mechanics and finite element for dynamic analysis of piezoelectric composite plates. Int J Solids Struct 1997; 34(3):359-78.

[19] Robbins Jr DH, Reddy JN. Modelling of thick composites using a layerwise laminated theory. Int J Numer Meth Eng 1993;36:655-77.

[20] Muller P, Touratier M. Dispersion of longitudinal waves in a rectangular transversely isotropic wave-guide. Wave Motion 1981;3:181-202.

[21] S. Wolfram, The Mathematica book, 4th ed., Mathematica version 4. Cambridge University Press, 1999. 